\title{
DIFFERENCES AMONG THE PERCEPTIONS OF ORTHODONTISTS, PROSTHODONTISTS AND CONSERVATIVE DENTISTRY SPECIALISTS REGARDING BUCCAL CORRIDOR WIDTHS
}

\author{
Muhammad Azeemª, Ali Razab, Rashid Mahmood ${ }^{c}$, Erum Behroz ${ }^{\mathrm{d}}$ \\ ${ }^{a}$ Assistant Professor, Department of Orthodontic, De'Montmorency College of Dentistry, Lahore. \\ ${ }^{\mathrm{b}}$ Assistant Professor, Department of Orthodontic, Akhter Saeed Dental College Lahore. \\ 'Senior Demonstrator/Consultant, Department of Orthodontic, CMH Dental Institute, Lahore. \\ ${ }^{\mathrm{d} A s s i s t a n t ~ P r o f e s s o r, ~ D e p a r t m e n t ~ o f ~ O r t h o d o n t i c, ~ S i n d h ~ I n s t i t u t e ~ o f ~ O r a l ~ H e a l t h ~ S c i e n c e s, ~ J i n n a h ~ S i n d h ~ M e d i c a l ~}$ \\ University, Karachi Pakistan.
}

\begin{abstract}
:
BACKGROUND \& OBJECTIVE: Buccal corridor width is related to smile attractiveness. The objective of the study was to find out the optimal ranges of smile attractiveness on basis of different amounts of buccal corridor widths, as judged by Pakistani orthodontists, prosthodontists and conservative dentistry specialists (CDS).

METHODOLOGY: Present cross-sectional study was conducted from June 2016 to June 2017. The anterior smiling image of a female model was edited to be converted to 10 images displaying different amounts of buccal corridor width. After informed consent from the subjects, different buccal corridor widths images were created followed by measurement of smile attractiveness on a visual analogue scale by 30 evaluators in three groups. Group-I consisted of 10 CDS, group-II consisted of 10 prosthodontists and group-III consisted of 10 orthodontists. The data were collected and scores of smile attractiveness were presented in form of mean and standard deviation for each group. The differences in the three groups regarding perception of smile attractiveness were found out using ANOVA test and Turkey's post-hoc test was used for multiple comparisons.

RESULTS: For group-I, the highest scoring was obtained by image showing $10 \%$ buccal corridor width. In group-II, the highest score was by image showing $14 \%$ buccal corridor width, whereas in group-III, the highest scoring was showing $12 \%$ buccal corridor width. The differences found were statistically insignificant.
\end{abstract}

CONCLUSION: Dental specialty does not affect perception of smile attractiveness. Buccal corridor width greater than $14 \%$ should be avoided.

KEYWORDS: Perception, Smile, Buccal corridors, Orthodontist perception.

How to cite this:

doi: https://doi.org/10.37723/jumdc.v11i4.432

Azeem M, Raza A, Mahmood R, Behroz E. DIFFERENCES AMONG THE PERCEPTIONS OF ORTHODONTISTS, PROSTHODONTISTS AND CONSERVATIVE DENTISTRY SPECIALISTS REGARDING BUCCAL CORRIDOR WIDTHS. jumdc. 2021;11(4):44-50.

doi: https://doi.org/10.37723/jumdc.v11i4.432

This is an Open Access article distributed under the terms of the Creative Commons Attribution License (http://creativecommons.org/licenses/by/4.0), which permits unrestricted use, distribution, and reproduction in any medium, provided the original work is properly cited. 


\section{INTRODUCTION:}

There are three types of esthetics: macroesthetics, micro-esthetics, and, miniesthetics ${ }^{[1]}$. Mini-esthetics is also known as smile analysis. Smile analysis deals with study of teeth-lip relations at rest and during smiling. There are several components of miniesthetics: interlabial gap, incisal inclinations, occlusal cant, incisal show at rest and smile, gingival show, philtrum and commissural height, height and width proportions, midlines, smile arc, smile symmetry, arch form and buccal corridors ${ }^{[2-3]}$.

Buccal corridors are lateral spaces on smiling, between the facial surfaces of maxillary premolars and inner side of $\operatorname{lips}^{[4]}$. There are different factors that affect buccal corridors: arch form, arch width, inclination of buccal teeth, anterio-posterior position of arches, vertical growth, and circum-oral soft tissues ${ }^{[5-7]}$. Previously limited studies have been conducted to find out the preferences of dental specialists in rating smile attractiveness based on different buccal corridor widths ${ }^{[8-11]}$. According to the recently conducted systematic review it was found that there is no general consensus about optimal buccal corridor width ${ }^{[12]}$. Few studies reported that decreased buccal corridor widths were perceived to be more attractive, while other studies found no link of buccal corridor widths and attractiveness of smile $e^{[13-15]}$.

Opinion of all the dental specialists should be of immense value while orthodontic treatment planning of patients with disturbed smile esthetics. To our knowledge, this is the first study to find out the optimal ranges of smile attractiveness on basis of different amounts of buccal corridor widths, as judged by Pakistani orthodontists, prosthodontists and conservative dentistry specialists (CDS). Following this rationale the objective of present study was to find out the optimal ranges of female smile attractiveness on basis of different amounts of buccal corridor widths, as judged by orthodontists, prosthodontists and CDS.

\section{METHODOLOGY:}

After taking informed consent and ethical approval, the present cross-sectional study was conducted from June 2016 to June 2017 at
Corresponding Author:

Dr. Muhammad Azeem

Assistant Professor, Department of Orthodontic, De'Montmorency College of Dentistry, Lahore.

'Email: dental.concepts@hotmail.com

Dental department of University Medical and Dental College, Faisalabad and De'Montmorency Dental College, Lahore. Inclusion criteria for the selected image was: A good quality and standardized anterior smiling view and anterior intra-oral view image of a female model. Subject had near normal occlusion according to Andrew's classification of occlusion.

Inclusion criteria for the evaluators were specialists of Conservative Dentistry, Prosthodontists and oral surgeons who were qualified in their respective fields and had at least 4 years postgraduate training experience in the respective fields.

The anterior smiling image of a female model was edited to convert into 10 images with different amounts of buccal corridor widths. The editing involved using adobe Photoshop software. First step was to do digital superimposition of anterior smiling image on intra-oral image. In the second step, oral cavity parts excluding lips were deleted from the smiling image to create the lip framework; this was followed by placement of intra-oral image behind this lip framework image. Third step was to convert this single image in to multiple buccal corridor width images, by editing the lateral dental parts while keeping frontal dental part constant. Ten different buccal corridor width images were created at following widths: $0 \%$, $2 \%, 4 \%, 6 \%, 8 \%, 10 \%, 12 \%, 14 \%, 16 \%$, and $18 \%$. The buccal corridor width was calculated using formula: Inter-commissural width minus width of dental arch, divided by intercommissural width multiplied by $100^{[16-17]}$.

This was followed by measurement of smile attractiveness of these 10 images by 30 evaluators in three groups. Group-I consisted of 10 CDS, group-II consisted of 10 prosthodontists and group-III consisted of 10 orthodontists. The evaluation of images was done using $10 \mathrm{~cm}$ visual analogue scale (from unattractive to very attractive $)^{[18,19]}$. 
In order to find out the reliability of the experts, intra-class correlation coefficients were calculated by requesting three evaluators from each group to rate the smile photographs again 07 days after the first scoring. The results of the intra-class correlation coefficients ranged from 0.79 to 0.88 ( $p$ 0.123). Smile attractiveness data was presented as scores of the mean and standard deviation (SD) of each group of experts (Table-I). The differences in between the three groups regarding perception of smile attractiveness were found out using One-way analysis of variance (ANOVA) test to assess how the groups rated each buccal corridor width value. Tuckey's Post-hoc analysis was used to see multiple comparisons (Table-II). The level of significance was set at $p<0.05$. Data analysis was undertaken using the Statistical Package for Social Science (version 20.0, SPSS Inc., Chicago, Illinois, USA).

\section{RESULTS:}

Response rate was $100 \%$. Total 30 evaluators (mean age $33.3 \pm 3.45$ ) participated in this research. Group-I consisted of $10 \mathrm{CDS}$ (mean age $32.4 \pm 3.12$ ), group-II consisted of 10 prosthodontists (mean age 34.8 \pm 3.21 ), and group 3 consisted of 10 orthodontists (mean age $33.1 \pm 3.10)$. The scores of smile attractiveness were presented in form of mean and standard deviation for each group of experts (Table-I). In group-I, the highest scoring (36.42 \pm 2.34$)$ for a buccal corridor width images were obtained by image showing $8 \%$ buccal corridor width. In a group-II, the highest scoring (36.45 \pm 8.12$)$ for a buccal corridor width images were obtained by image showing $12 \%$ buccal corridor width. In a group 3 , the highest scoring (35.55 \pm 8.24$)$ for a buccal corridor width images were obtained by image showing $10 \%$ buccal corridor width.

Table-II shows that differences were statistically insignificant among the scores of orthodontists, Prosthodontists and CDS regarding perception of smile attractiveness on basis of different amounts of buccal corridor widths.

Table-I: Smile attractiveness scores for different buccal corridor widths.

\begin{tabular}{|c|c|c|c|}
\hline $\begin{array}{l}\text { Buccal corridor } \\
\text { width }(\%)\end{array}$ & $\begin{array}{l}\text { Group-I (CDS)* } \\
(\text { mean } \pm \text { SD) } * * \\
\quad \mathbf{n}=10\end{array}$ & $\begin{array}{c}\text { Group-II } \\
\text { (Prosthodontists) } \\
\text { (mean } \pm \text { SD) } \\
n=10\end{array}$ & $\begin{array}{c}\text { Group-III } \\
\text { (Orthodontists) } \\
(\text { mean } \pm \text { SD) } \\
n=10\end{array}$ \\
\hline $0 \%$ & $32.12 \pm 6.44$ & $33.23 \pm 8.24$ & $34.32 \pm 8.86$ \\
\hline $2 \%$ & $28.21 \pm 6.78$ & $27.32 \pm 8.64$ & $29.22 \pm 8.33$ \\
\hline $4 \%$ & $28.15 \pm 5.21$ & $27.12 \pm 8.22$ & $29.55 \pm 8.33$ \\
\hline $6 \%$ & $28.17 \pm 7.86$ & $27.12 \pm 8.22$ & $29.33 \pm 8.51$ \\
\hline $8 \%$ & $36.42 \pm 2.34$ & $27.76 \pm 8.32$ & $29.87 \pm 8.89$ \\
\hline $10 \%$ & $31.64 \pm 8.77$ & $31.54 \pm 8.54$ & $35.55 \pm 8.24$ \\
\hline $12 \%$ & $31.22 \pm 4.44$ & $36.45 \pm 8.12$ & $33.54 \pm 8.21$ \\
\hline $14 \%$ & $24.66 \pm 5.98$ & $23.34 \pm 8.21$ & $25.34 \pm 8.12$ \\
\hline $16 \%$ & $25.82 \pm 9.32$ & $24.75 \pm 8.65$ & $26.55 \pm 8.11$ \\
\hline $18 \%$ & $25.00 \pm 5.85$ & $25.66 \pm 8.45$ & $27.22 \pm 8.43$ \\
\hline $20 \%$ & $26.32 \pm 5.22$ & $25.28 \pm 8.42$ & $26.22 \pm 8.21$ \\
\hline
\end{tabular}

*conservative dentistry specialists $* *$ standard deviation 
Table-II: Differences among the three groups.

\begin{tabular}{|c|c|c|c|}
\hline $\begin{array}{c}\text { Buccal corridor } \\
\text { width }(\%)\end{array}$ & $\begin{array}{c}\text { Group-I-II } \\
p \text {-value }\end{array}$ & $\begin{array}{c}\text { Group-I-III } \\
p \text {-value }\end{array}$ & $\begin{array}{c}\text { Group-II-III } \\
p \text {-value }\end{array}$ \\
\hline $0 \%$ & 0.063 & 0.071 & 0.072 \\
\hline $2 \%$ & 0.062 & 0.081 & 0.091 \\
\hline $4 \%$ & 0.072 & 0.082 & 0.080 \\
\hline $6 \%$ & 0.091 & 0.073 & 0.066 \\
\hline $8 \%$ & 0.080 & 0.074 & 0.063 \\
\hline $10 \%$ & 0.084 & 0.074 & 0.082 \\
\hline $12 \%$ & 0.077 & 0.072 & 0.077 \\
\hline $14 \%$ & 0.073 & 0.066 & 0.071 \\
\hline $16 \%$ & 0.061 & 0.063 & 0.081 \\
\hline $18 \%$ & 0.064 & 0.082 & 0.082 \\
\hline $20 \%$ & 0.066 & 0.077 & 0.097 \\
\hline
\end{tabular}

Group-I = CDS, Group -II = Prosthodontists, Group-III= Orthodontists Multiple comparisons is seen using post-hoc Tuckey's test ( $p$-value $<0.05$ was considered statistically significant).

\section{DISCUSSION:}

Numerous factors affect the foundation of esthetic beauty standards including culture. Although various studies from the past literature conducted on the role of buccal corridor and golden proportion assessing the smile attractiveness had been performed, there was no published paper comparing the perception of smile esthetics among the Pakistani dental specialists from various disciplines ${ }^{[20]}$.

Data from the previous research on people from diverse ethnicities and cultural values exhibited remarkable variation when defining the esthetic parameters. Surveys conducted over samples from different groups in detecting deviations from an ideal smile concluded that cultural disparity related to smile characteristics, undeniably exist ${ }^{[21-22]}$. It is important to consider the perception of dental specialists of relevant disciplines while planning orthodontic treatment for patients with distorted smile esthetics. While some dental specialists do not pay much attention to unnecessary correction of small asymmetries or dissimilarities assuming that most patients cannot detect them, others may consider it to be extremely desirable to treat the case according to the standard protocols ${ }^{[20]}$. Previously limited research has been conducted to find out optimal ranges of smile attractiveness on basis of different amounts of buccal corridor widths involving various dentistry specialties in general and in Pakistan in particular. Following this rationale, the objective of present study was to find out the optimal ranges of female smile attractiveness on basis of different amounts of buccal corridor widths. In the present study, only photographs of female model were included, the primary reason for including single gender was inconsistency in the results for using both male and female population photographs. Moreover, previous studies reported inconclusive findings in terms of documenting differences for the values of attractiveness using single variable as it was driven by other variables too ${ }^{[17-18]}$.

In the present study, visual analogue scale was used to rate the attractiveness of smile. Visual analogue scale is one of the most commonly used tools to rate pain of orthodontic patients and has been shown to be a valid and reliable method ${ }^{[19,20]}$. Various authors have also used this scale for the rating of smile attractiveness $^{[17]}$. This scale was used in the present study because it is simple and quick with reproducible results ${ }^{[18-19]}$.

Gender is not important in rating of buccal corridor width preferences or smile 
attractiveness ${ }^{[23-24]}$. The results of this study showed that differences were insignificant among the scores of orthodontists, prosthodontists and CDS regarding perception of smile attractiveness on the basis of buccal corridor widths. This is in contrast with the findings of study, where differences were found in rating of buccal corridor width preferences or smile attractiveness between the dental specialists ${ }^{[16]}$. This may be due to the difference in knowledge of dental specialists regarding smile esthetics.

In the present study, all the dental specialists judged buccal corridor width $>14 \%$ less attractive. Study by Oz et al. showed that dental specialists judged buccal corridor width $>16 \%$ less attractive ${ }^{[16]}$. This also showed that dental specialists should keep in mind that small alterations in buccal corridor width amount can significantly affect the perception of smile attractiveness.

The findings of this study showed that all the dental specialists preferred narrow buccal corridors over wider buccal corridors. This is in agreement with the findings of past studies where evaluators preferred narrow buccal corridors over wider buccal corridors, ${ }^{[18]}$ but in contrast with the findings of studies by Roden et al., where evaluators preferred narrow buccal corridors over wider buccal corridors ${ }^{[25]}$.

Limitations of this study were small sample size in the form of small number of specialists in each of the three groups (10 specialist each group), rating on female images only, and lack of standardization of images for evaluating other factors related to smile attractiveness. However, within these limitations, findings showed that the type of dental specialty does not affect perception of smile attractiveness. Buccal corridor width greater than $14 \%$ should be avoided. Further large-scale studies are suggested to find out the optimal ranges of male and female smile attractiveness on basis of different amounts of buccal corridor widths, as judged by orthodontists, prosthodontists and CDS.

\section{CONCLUSION:}

The types of dental specialties do not affect perception of smile attractiveness. Buccal corridor width greater than $14 \%$ should be avoided.

\section{ACKNOWLEDGEMENT:}

This study would not have been possible without the positive feedback of various dental departments of both the institutes.

CONFLICT OF INTEREST: All authors disclose no conflict of interest.

\section{GRANT SUPPORT \& FINANCIAL DISCLOSURE: None}

\section{REFERENCES:}

1. Cotrim ER, VasconcelosJúnior ÁV, Haddad AC, Reis SA. Perception of adults' smile esthetics among orthodontists, clinicians and laypeople. Dental Press Journal of Orthodontics. $2015 ; 20(1): 40-44$. Doi: $10.1590 / 2176-9451.20 .1 .040$ 044.oar

2. Sarver DM. Orthodontics \& esthetic dentistry: mission possible! A Broader Approach to Interdisciplinary Esthetic Treatment. Journal of Cosmetic Dentistry. 2016;31(4):14-26.

3. Katiyar S, Gandhi S, Sodawala J, Anita G, Hamdani S, Jain S. Influence of symmetric and asymmetric alterations of maxillary canine gingival margin on the perception of smile esthetics among orthodontists, dentists, and laypersons. Indian Journal of Dental Research. 2016;27(6):586.

4. Nimbalkar S, Oh YY, Mok RY, Tioh JY, Yew KJ, Patil PG. Smile attractiveness related to buccal corridor space in 3 different facial types: A perception of 3 ethnic groups of Malaysians. The Journal of Prosthetic Dentistry. 2018;120(2):252-256. Doi:10.1016/j.prosdent.2017.10.021

5. Nyukhtikov M, Morozov E, Prokoshev V, Likhacheva O, Lapshin A, Sterental RM, et al. Buccal corridor assessment and computation. United States patent application US 15/998,883. 2019.

6. Gaikwad S, Kaur H, Vaz AC, Singh B, Taneja $L$, Vinod KS, et al. influence of smile arc and buccal corridors on facial attractiveness: A Cross-sectional Study. Journal of Clinical and Diagnostic Research: 2016;10(9): 
ZC20. Doi: $10.7860 / J C D R / 2016 / 19013$. 8436.

7. Niaki EA, Arab S, Shamshiri A, Imani MM. The effect of the buccal corridor and tooth display on smile attractiveness. Australian Orthodontic Journal. 2015;31(2):195.

8. Lacerda-Santos R, Pereira TB, Pithon MM. Esthetic perception of the buccal corridor in different facial types by laypersons of different ages. Bioscience Journal. 2015;31(4). Doi: https://doi.org/10. 14393/BJ-v31n4a2015-28654

9. Najafi HZ, Oshagh M, Azizi M. Esthetic effect of the buccal corridor size and amount of tooth-gingival display on smile attractiveness in two student population. Journal of Dental Medicine. 2015;28 (1):57-67.

10. Sikandar $H$, Adil S. Perception of an esthetical smile in relation to the buccal corridor. Pakistan Orthodontic Journal. 2018;10(2):87-90.

11. Mollabashi V, Abolvardi M, Akhlaghian M, Ghaffari MI. Smile attractiveness perception regarding buccal corridor size among different facial types. Dental and Medical Problems. 2018;55(3):305-312.

12. Janson G, Branco NC, Fernandes TM, Sathler R, Garib D, Lauris JR. Influence of orthodontic treatment, midline position, buccal corridor and smile arc on smile attractiveness. Angle Orthodontist. 2011; 81: 153-161.

13. Parekh S, Fields HW, Beck FM, Rosenstiel SF. The acceptability of variations in smile arc and buccal corridor space. Orthodontics and Craniofacial Research. 2007; 10:15-21.

14. Ioi $H$, Nakata $S$, Counts AL. Effects of buccal corridors on smile esthetics in Japanese. Angle Orthodontist. 2009;79: 628-633.

15. McNamara L, McNamara Jr JA, Ackerman MB, Baccetti T. Hard-and soft-tissue contributions to the esthetics of the posed smile in growing patients seeking orthodontic treatment. American Journal of Orthodontics and Dentofacial Orthopedics. 2008;133(4):491-499.

16. Öz AA, Akdeniz BS, Canli E, Çelik S. Smile attractiveness: differences among the perceptions of dental professionals and laypersons. Turkish Journal of Orthodontics. 2017;30(2):50-55.Doi: 10.5152/TurkJOrthod.2017.17021

17. Tovée MJ, Maisey DS, Vale ELE, Cornelissen PL. Characteristics of male attractiveness for women. Lancet. 1999; 353:1500. Doi: 10.1016/S0140-6736(99) 00438-9

18. Azeem M, Khan DI, Jamal F, Ali S, Fatima $M$, Hayat $M B$, et al. Chewing gum for pain control following orthodontic separator placement. Journal of the World Federation of Orthodontists. 2018;7(3): 102-105. Doi:10.1016/j.ejwf. 2018. 07.002

19. Al Taki A, Khalesi M, Shagmani M, Yahia I, Al Kaddah F. Perceptions of altered smile esthetics: A comparative evaluation in orthodontists, dentists, and laypersons. International Journal of Dentistry. 2016. Doi:10.1155/2016/7815274

20. Saffarpour A, Ghavam M, Saffarpour A, Dayani R, Fard MJ. Perception of lay people and dental professionals of smile esthetics. Journal of Dentistry. 2016;13(2):85-91. PMID: 27928236

21. Alhajj MN, Ariffin Z, Celebic A, Alkheraif AA, Amran AG, Ismail IA. Perception of orofacial appearance among laypersons with diverse social and demographic status. PLos One. 2020;15(9):e0239232.

22. Jain RK, Dinesh S, Priya A, Kumar MP. Comparison of perception of smile by orthodontists and general dental practitioners-A questionnaire study. Drug Invention Today. 2019;12(2).

23. Martin AJ, Buschang PH, Boley JC, Taylor RW, McKinney TW. The impact of buccal corridors on smile attractiveness. The European Journal of Orthodontics. 2007 ;29(5):530-537. Doi:10.1093/ejo/ cjm063

24. Gracco A, Cozzani M, D'Elia L, Manfrini M, Peverada C, Siciliani G. The smile buccal corridors: aesthetic value for dentists and laypersons. Progress in Orthodontics. $2006 ; 7(1): 56-65$.

25. Roden-Johnson D, Gallerano R, English J. The effects of buccal corridor spaces and arch form on smile esthetics. American Journal of Orthodontics and Dentofacial Orthopedics. 2005;127(3):343-350. 


\section{Author's Contribution}

Muhammad Azeem: Data analysis, Reviewed the manuscript.

Ali Raza: Designed the study, collected the data.

Rashid Mahmood: Data collection \& Analysis.

Erum Behroz: Manuscript writing, Data collection.

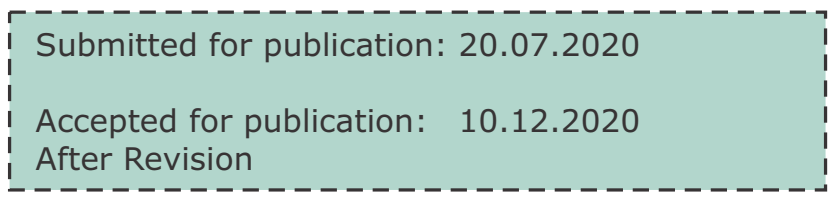

